\title{
Strand-asymmetric endogenous Tetrahymena small RNA production requires a previously uncharacterized uridylyltransferase protein partner
}

\author{
KRISTIN BENJAMIN TALSKY and KATHLEEN COLLINS ${ }^{\mathbf{1}}$ \\ Department of Molecular and Cell Biology, University of California, Berkeley, California 94720-3200, USA
}

\begin{abstract}
Many eukaryotes initiate pathways of Argonaute-bound small RNA (sRNA) production with a step that specifically targets sets of aberrant and/or otherwise deleterious transcripts for recognition by an RNA-dependent RNA polymerase complex (RDRC). The biogenesis of 23- to 24-nt sRNAs in growing Tetrahymena occurs by physical and functional coupling of the growth-expressed Dicer, Dcr2, with one of three RDRCs each containing the single genome-encoded RNA-dependent RNA polymerase, Rdr1. Tetrahymena RDRCs contain an active uridylyltransferase, either Rdn1 or Rdn2, and Rdn1 RDRCs also contain the Rdf1 and Rdf2 proteins. Although Rdn2 is nonessential and RDRC-specific, Rdn1 is genetically essential and interacts with a non-RDRC protein of $124 \mathrm{kDa}$. Here we characterize this 124-kDa protein, designated RNA silencing protein 1 (Rsp1), using endogenous locus tagging, affinity purification, and functional assays, as well as gene-knockout studies. We find that Rsp1 associates with Rdn1-Rdf1 or Rdn1-Rdf2 subcomplexes as an alternative to Rdr1, creating Rsp1 complexes (RSPCs) that are physically separate from RDRCs. The uridylyltransferase activity of Rdn1 is greatly reduced in RSPCs compared with RDRCs, suggesting enzyme regulation by the alternative partners. Surprisingly, despite the loss of all known RDRC-generated classes of endogenous sRNAs, RSP1 gene knockout was tolerated in growing cells. A minority class of Dcr2-dependent sRNAs persists in cells lacking Rsp1 with increased size heterogeneity. These findings bring new insights about the essential and nonessential functions of RNA silencing in Tetrahymena, about mechanisms of endogenous small interfering RNA production, and about the roles of cellular uridylyltransferases.
\end{abstract}

Keywords: RNA silencing; RNA-dependent RNA polymerase; poly(U) polymerase; Piwi RNP

\section{INTRODUCTION}

Among the cellular RNA recognition mechanisms that compete to determine the fate of mRNAs and mRNA-like transcripts, the specificity for initiating endogenous RNA silencing is among the least understood. Production of some endogenous small RNAs (sRNAs) from annealed transcripts is thought to be initiated by double-stranded RNA (dsRNA) binding to Dicer complexes (Carthew and Sontheimer 2009; Ketting 2011). However, in many eukaryotes, the abundant endogenous sRNAs are dependent on active rather than passive generation of dsRNA mediated by an RNA-dependent RNA polymerase (Rdr). Some Rdr-dependent dsRNA synthesis occurs secondary to original transcript targeting for RNA silencing, for example, the production of some types of small

\footnotetext{
${ }^{1}$ Corresponding author

E-mail kcollins@berkeley.edu

Article published online ahead of print. Article and publication date are at http://www.rnajournal.org/cgi/doi/10.1261/rna.033530.112.
}

interfering RNA (siRNA) in Arabidopsis thaliana and Dicerindependent amplification of siRNAs in Caenorhabditis elegans (Vazquez et al. 2004; Pak and Fire 2007; Sijen et al. 2007). Rdrdependent dsRNA synthesis can also be specified by tethering the enzyme to chromatin, as established in Schizosaccharomyces pombe in mechanistic detail (Grewal 2010; Moazed 2011). In addition to these functions in perpetuating siRNA production, an Rdr can act upstream of any other known pathway step to initiate the silencing of mRNA-like transcripts (Voinnet 2008; Ketting 2011; Thivierge et al. 2012). The principles that underlie the cellular specificity of transcript recognition by an Rdr are unknown, but they must provide for both target generality (to be evolutionarily useful) and also target specificity (to avoid selecting a functional RNA for self-perpetuating silencing). It seems likely that the varied subunits of cellular Rdr complexes (RDRCs) contribute to the specialization of Rdr function in vivo, restricting the general activity of $\mathrm{Rdr}$ enzymes that is observed on single-stranded RNA templates in vitro (Voinnet 2008; Talsky and Collins 2010). 
Ciliated protozoa have a large complexity of RNA silencing machinery relative to other single-celled organisms, including numerous Piwi proteins that are the exclusive Argonaute family members encoded by ciliate genomes sequenced to date (Couvillion et al. 2009; Bouhouche et al. 2011). In addition to conjugationspecific silencing pathways that induce the transient heterochromatin formation and DNA elimination necessary for sexual reproduction, additional pathways produce sRNAs constitutively during culture expansion by vegetative growth (Duharcourt et al. 2009; Mochizuki 2012). The Tetrahymena Dicer, Dcr2, and the single genome-encoded Rdr, Rdr1, function coordinately to generate multiple classes of 23- to 24-nucleotide (nt) sRNAs in vegetatively growing cells (Lee and Collins 2006; Couvillion et al. 2009). Pseudogene transcripts, arising from open reading frames with downstream genome-encoded polyadenosine tracts, generate unphased antisense sRNAs. Other mRNA-like transcripts with potential to form a long $3^{\prime}$ hairpin generate a phased register of strand-specific antisense sRNAs, which map in clusters upstream of the putative hairpin. Other, less abundant classes of sRNAs derive from repetitive regions of the genome, including telomeres, or from sites of convergent transcription that have potential to produce dsRNA by annealing rather than Rdr-mediated synthesis.

Distinct Tetrahymena RDRCs (Fig. 1A) direct sRNA biogenesis from different types of initiating transcript, with only the RDRC-synthesized strand sRNAs stabilized by assembly with a Tetrahymena Piwi (Twi) protein. Tetrahymena RDRCs share the Rdr1 subunit but assemble either $\operatorname{Rdn} 1$ or Rdn2, one of two paralogous proteins with similar uridylyltransferase activity in vitro (Lee and Collins 2007; Lee et al. 2009; Talsky and Collins 2010). Rdn1 RDRCs also recruit Rdf1 and/or Rdf2, two other paralogous proteins that lack any known protein domain (Lee and Collins 2007; Lee et al. 2009). Independent of these differences in subunit composition, each RDRC copurifies directly associated Dcr2 enzyme in low-salt wash conditions (Lee and Collins 2007; Lee et al. 2009). Although Dcr2, Rdr1, and Rdn1 are genetically essential for vegetative growth, each of the other individual RDRC subunits is not (Lee and
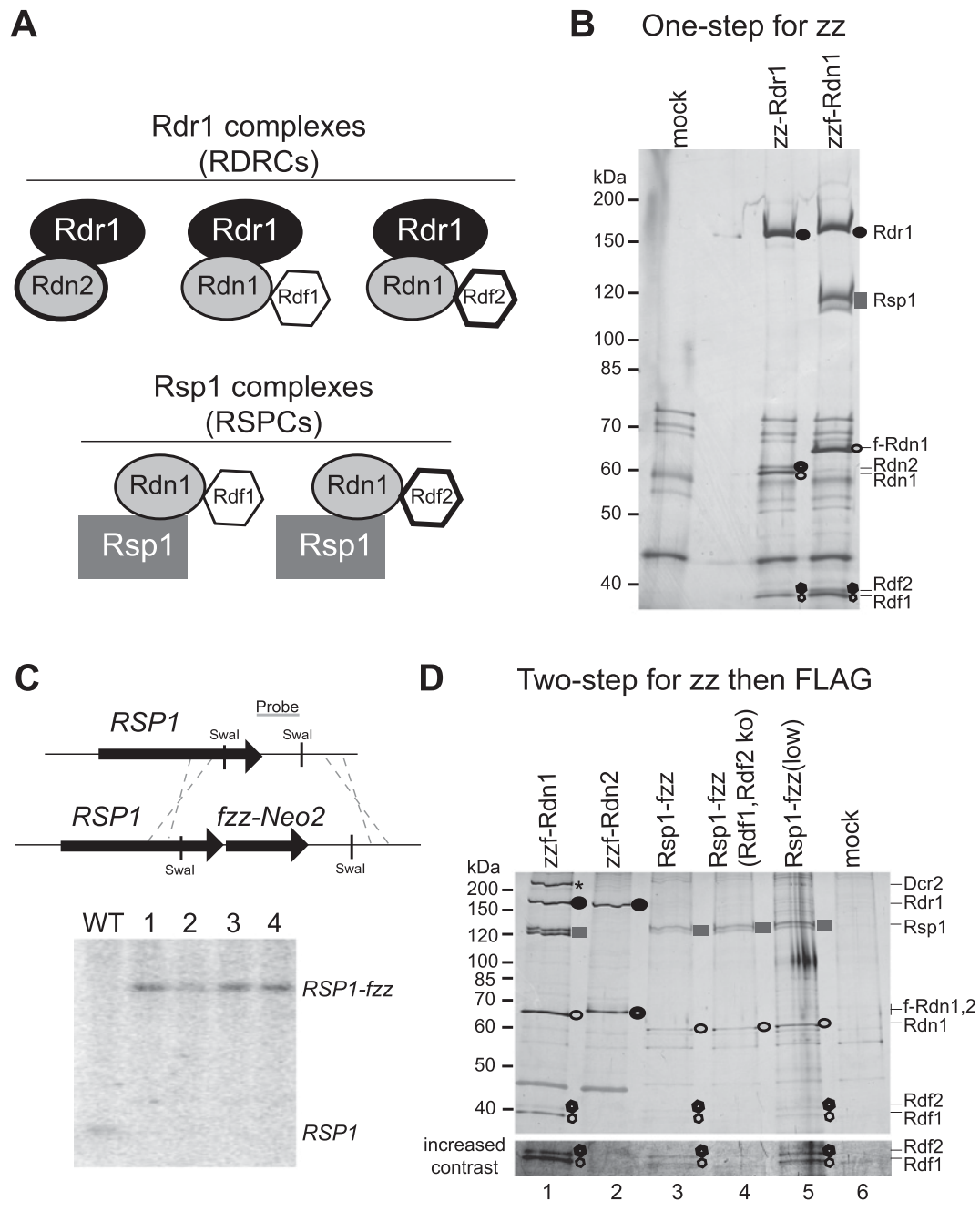

FIGURE 1. Subunit sharing by RDRPs and RSPCs. (A) Schematics of the sets of protein complexes containing Rdr1 or Rsp1 are shown, based on previous studies and studies described here. The same symbol shapes and fill densities are used to annotate protein identities in the SDS-PAGE gels; RDRC-associated Dcr2 is annotated in SDS-PAGE gel panels as an asterisk. (B) SDS-PAGE and silver staining were used to detect the subunit content of complexes purified from cell extracts of the indicated tagged protein expression strain or from wild-type cells lacking a tagged protein (mock). Complexes were purified by IgG agarose binding and TEV protease elution. (C) A C-terminal fzz tag followed by a Neo2 drugresistance cassette was integrated at the RSP1 locus as schematized. Locus assortment was analyzed by genomic DNA digestion with the indicated restriction enzyme and Southern blot detection of differentially sized DNA fragments (the genomic region used as probe is shown). (D) SDS-PAGE and silver staining were used to detect the subunit content of complexes purified from cell extracts of the indicated tagged protein expression strain or from wild-type cells lacking a tagged protein (mock). Complexes were purified by sequential IgG agarose binding, TEV protease elution, Flag antibody binding, and Flag peptide elution. One purification, Rsp1-fzz(low), was washed only in low-salt buffer.
Collins 2006, 2007; Lee et al. 2009; Malone et al. 2005; Mochizuki and Gorovsky 2005). Pseudogene-derived sRNAs are absent in cells lacking Rdn2, phased-cluster sRNAs are absent in cells lacking Rdf2, and less abundant classes of sRNAs are affected in more than one of the individual RDRC subunit knockout strains (Couvillion et al. 2009; Lee et al. 2009). At the cellular level, Rdf1 or Rdf2 knockout gives rise to a partially penetrant cell-division phenotype, while 
loss of Rdf1 or Rdn2 blocks the progression of nuclear events in conjugation (Lee et al. 2009). These findings imply that each RDRC has distinct function(s) in vivo, but in vitro each RDRC has similar biochemical activities and Rdr1 itself is sufficient for processive dsRNA synthesis (Lee et al. 2009; Talsky and Collins 2010). The Rdn subunit of an RDRC can act before Rdr1 to add mono- or oligo-uridine to a singlestranded RNA 3' end, which promotes use of the RNA 3' end in the obligate back-priming mechanism of Tetrahymena Rdr1 initiation (Talsky and Collins 2010). Template tailing prior to dsRNA synthesis affects the register of cotranscriptional cleavage by Dcr2 (Talsky and Collins 2010).

Additional understanding of the specialization and regulation of RDRCs is needed to elucidate how particular mRNA and mRNA-like transcripts are selected for commitment to dsRNA synthesis in vivo. Our previous studies showed that although Rdn1 and Rdn2 have similar catalytic activity, only Rdn1 is essential for culture growth (Lee et al. 2009). Rdn1 but not Rdn2 interacts with an uncharacterized protein of $124 \mathrm{kDa}$, which we designate here as RNA silencing protein 1 (Rspl). Genetic, molecular, and biochemical assays reveal that Rsp1 is required for strand-specific antisense sRNA production by all RDRCs and suggest new steps of sRNA biogenesis in pathways with Rdr-dependent initial dsRNA synthesis.

\section{RESULTS}

\section{Rsp1 interacts with RDRC subunits but not RDRCs}

To further our understanding of functional distinctions among Tetrahymena RDRCs (Fig. 1A), we characterized the $124 \mathrm{kDa}$ protein detected in association with Rdn1 (Lee et al. 2009). By using strains created previously (Lee and Collins 2007; Lee et al. 2009), we confirmed that the 124$\mathrm{kDa}$ protein was not evident in an IgG agarose purification of Rdr1 tagged with tandem Protein A domains (zz-Rdr1) but was abundant in a parallel purification of $\mathrm{Rdn} 1$ with tandem Protein A domains and triple Flag peptide (zzfRdn1). Compared to a mock purification from wild-type cell extract, Rdr1 specifically enriched $\operatorname{Rdn} 1, \operatorname{Rdn} 2, \operatorname{Rdf} 1$, and Rdf2; Rdn1 enriched Rdr1, Rdf1, Rdf2, and a doublet of $\sim 124 \mathrm{kDa}$ (Fig. 1B). Based on peptide sequences from mass spectrometry (Lee et al. 2009), we cloned the cDNA encoding the $124-\mathrm{kDa}$ protein designated here as Rsp1. Despite its length, no known protein domain was detected in Rsp1 by homology. We tagged Rsp1 at its C terminus with a triple Flag peptide followed by tandem Protein A domains (Rsp1-fzz), using targeted integration at the endogenous gene locus (Fig. 1C). Based on Southern blot hybridization of post-selection clonal cell lines, the transgene encoding Rsp1-fzz completely replaced the wild-type RSP1 locus (Fig. 1C).

We performed two-step affinity purification of RDRCs and Rsp1 complexes (RSPCs) from extracts of cells expressing tagged proteins, using wild-type cell extract as a mock purification control. As expected, in addition to Rdr1 and Dcr2, zzf-Rdn1 copurified Rsp1 and the Rdf subunits that are not associated with zzf-Rdn2 (Fig. 1D, lanes 1,2; note that some Dcr2 was retained through the purification of Rdn1 RDRCs even with our standard $200 \mathrm{mM} \mathrm{NaCl}$ saltwash conditions). In comparison, Rsp1-fzz did not copurify Rdr1 or Dcr2 but did copurify proteins that comigrate with Rdf1 and Rdf2 (Fig. 1D, lane 3). These associated proteins were lost from Rsp1-fzz purifications in double gene knockout background lacking Rdf1 and Rdf2 (Southern blot data not shown), demonstrating their identity (Fig. 1D, lane 4). Even gentle low-salt wash conditions that stabilize RDRC-Dcr2 association did not reveal Rdr1 or Dcr2 as an interaction partner of Rsp1 (Fig. 1D, lane $5)$. We conclude that RDRCs and RSPCs share Rdn 1 and the Rdfs but are mutually exclusive for Rdr1 or Rsp1 (Fig. 1A).

The purifications described above and in previous studies do not resolve whether the related Rdf proteins are mutually exclusive components of an RDRC or RSPC, as are the related $\mathrm{Rdn} 1$ and $\mathrm{Rdn} 2$, or can be present together within a single RDRC. To address this question, we tagged the $\mathrm{C}$ terminus of Rdf1, Rdf2, and Rdn2 expressed at their endogenous loci to generate fusion proteins with a triple Flag tag and GFP (the fg tag). Based on Southern blot hybridization of post-selection clonal cell lines, the transgene encoding each fusion protein replaced the expressed wildtype locus (Fig. 2A). Perhaps due to diffuse cytoplasmic distribution, endogenous subunit expression levels were insufficient to detect subcellular concentration of the GFP signal (data not shown). Affinity purifications of the fgtagged proteins using Flag antibody resin recovered the expected specificity of $\mathrm{Rdr} 1, \mathrm{Rdn} 1$, and Rsp1 association, without Rdf1 copurification of Rdf2 or vice versa (Fig. 2B, cf. lanes 1-4 and lane 5). Thus, like Rdn1 and Rdn2, Rdf1 and Rdf2 are alternative subunits of an RDRC or RSPC (Fig. 1A).

\section{Rdn1 activity differs in RDRC or RSPC context}

Rdn1 and Rdn2 have robust uridylyltransferase activity on single-stranded RNA substrates when assayed as part of a purified RDRC (Lee et al. 2009; Talsky and Collins 2010). Rdn activity as a uridylyltransferase does not depend on Rdr1 activity in vitro, because it is unaffected in RDRCs purified with the catalytic-dead Rdr1(D1004A). To investigate whether $\mathrm{Rdn} 1$ retains uridylyltransferase activity in RSPC context, we used our standard Rdn assay conditions with a 79-nt single-stranded RNA and radiolabeled $\alpha^{32} \mathrm{P}$ UTP (Talsky and Collins 2010). Products were resolved by formamide-urea polyacrylamide gel electrophoresis (PAGE) to prevent potential RNA secondary structure formation. Compared to a mock purification from wild-type cell extract (Fig. 3A, lane 1), product radiolabeling occurred specifi- 


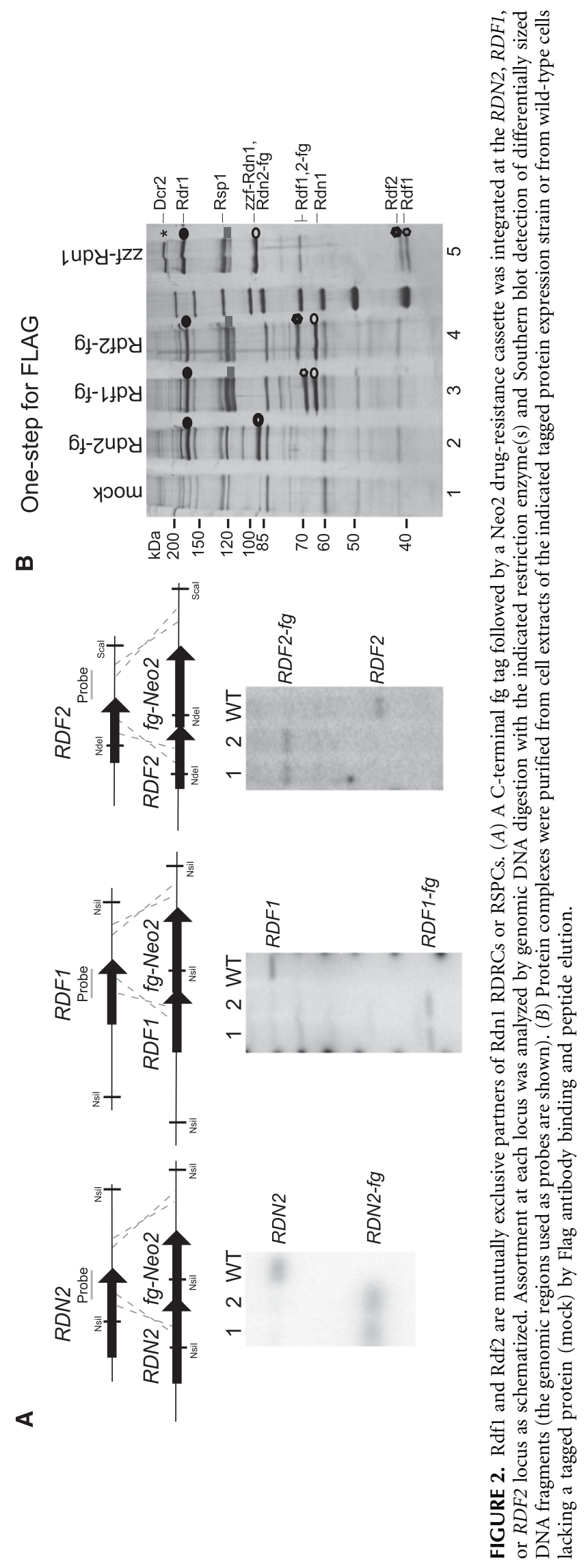



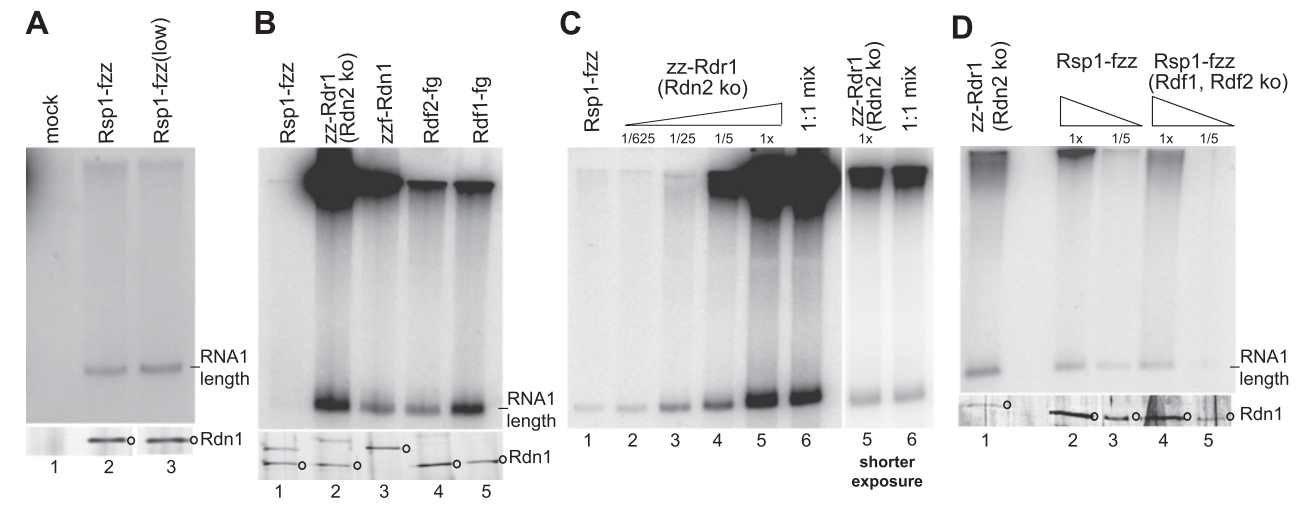

FIGURE 3. Rdn1 activity is influenced by association with Rdr1 or Rsp1. (A-D) Protein complexes were purified from extracts of wild-type cells or the indicated tagged protein expression strain by binding to IgG agarose and elution with TEV protease. One purification, Rsp1fzz(low), was washed only in low-salt buffer. Rdn1 protein content was normalized by silver staining after SDS-PAGE (bottom panels), prior to activity assay by radiolabeled UTP addition to the single-stranded RNA1. Polyuridine addition products at the top of the gel are resolved from the less processively elongated products migrating at approximately input template length. The same purifications used in $B$ were also used for dilution or mixture in $C$.

cally in purifications of Rsp1-fzz that used either the typical $200 \mathrm{mM} \mathrm{NaCl}$ or gentle $50 \mathrm{mM} \mathrm{NaCl}$ wash conditions (Fig. 3A, lanes 2,3, respectively). As characterized previously for RDRCs (Talsky and Collins 2010), RSPCs produced both near-template length and longer polyuridine products. However, when normalized by the input of Rdn1 (Fig. 3, lower panels), RSPCs had much less specific activity than RDRCs. Compared to Rdn1 RDRCs purified using zz-Rdr1 in an Rdn2 knockout background (Lee et al. 2009), the uridylyltransferase products of RSPCs were dramatically reduced (Fig. 3B, lanes 1,2). This difference in activity was consistent across assays using different RNA substrates (data not shown). Mixtures of RSPCs and RDRCs copurified by tagged Rdn1, Rdf1, or Rdf2 catalyzed the expected intermediate level of activity normalized to total input Rdn1 (Fig. 3B, lanes 3-5).

We next assayed the uridylyltransferase activity of RSPCs in comparison to a serial dilution of Rdn1 RDRCs (Fig. 3C). RDRC dilution by about 1000 -fold brought the level of uridylyltransferase activity to match that of RSPCs with normalized Rdn1 content (Fig. 3C, cf. lanes 2-5 and lane 1). As a control for RSPC copurification of a nonspecific Rdn1 inhibitor, the mixing of purified RSPCs and RDRCs did not inhibit Rdn1 activity (Fig. 3C, shorter exposure of lanes 5,6). Purification of Rsp1-fzz from extracts of cells lacking Rdf1 and Rdf2 did not obviously change RSPC uridylyltransferase activity (Fig. 3D), consistent with the lack of Rdf subunit influence on the in vitro uridylyltransferase activity of an RDRC (Lee et al. 2009). The length profile of product synthesis by RSPCs and RDRCs was not notably different; considering the high specific activity of the polyuridine addition products, shorter mono- or oligouridine addition products are predominant. Formally it remains possible that Rsp1 itself catalyzes uridylyltransferase activity, but because catalytic-dead Rdn1 is highly toxic for Tetrahymena growth (Lee et al. 2009), it is not possible to assay Rsp1 activity in an RSPC with inactive Rdn1.

\section{Cells lacking Rsp1 are viable for growth but compromised in sexual reproduction}

To address the in vivo function of Rsp1, including its potential influence on sRNA biogenesis, we targeted the endogenous RSP1 locus for disruption with a cassette conferring resistance to blasticidin (see Materials and Methods). In parallel we targeted the RDN1 locus for disruption with the same cassette. Based on Southern blot hybridization of post-selection clonal cell lines, the macronuclear chromosome with disruption of RSP1 could completely replace the chromosome containing wild-type RSP1 in two strains of complementary mating type (CU522 clonal strain 4 and SB210 clonal strain 4 were used for subsequent studies) (Fig. 4A). Thus, as is true for its associated Rdf1 and Rdf2 subunits, Rsp1 is not essential for vegetative growth. In contrast, consistent with previous results (Lee et al. 2009), the wild-type RDN1 locus could not be fully replaced (data not shown).

Conjugating cell pairs that lack parental Rdn2 or Rdf1 fail to progress through the nuclear differentiation events that produce new macronuclei (Lee et al. 2009). We therefore addressed whether cells lacking parental Rsp1 also have a conjugation defect. Mated wild-type SB210 and CU522 cells formed stable pairs and by $9 \mathrm{~h}$ post-mixing generated two new developing macronuclei and two new nuclei with micronuclear content positioned anterior to the strongly DAPI-staining, posterior-localized parental macronucleus (Fig. 4B, top panels; the anterior ends of the paired cells are indicated with arrowheads). In contrast, although stably paired and normal compared with wild-type cells for $\sim 4 \mathrm{~h}$ post-mixing, mated SB210 and CU522 cells with macronu- 
A

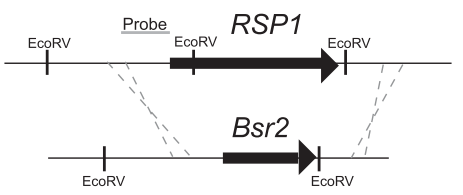

CU522

SB210

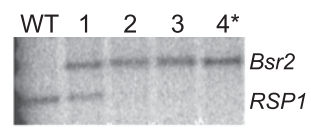

WT $1 \quad 2 \quad 3 \quad 4^{*}$

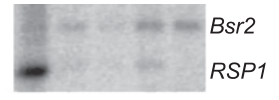

B WT $\times$ WT
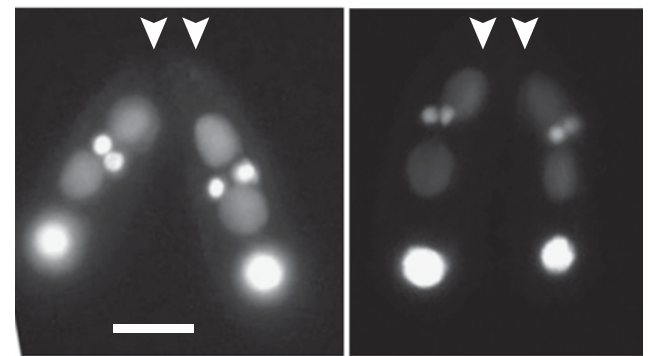

ko $\mathrm{x}$ ko

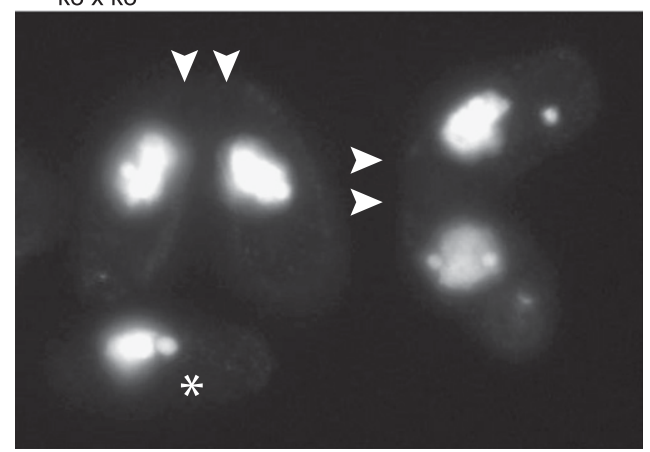

FIGURE 4. Loss of Rsp1 is tolerated in vegetative growth but impedes conjugation. (A) A drug-resistance cassette was integrated at the RSP1 locus as schematized in strains with different mating types. Assortment was analyzed by genomic DNA digestion with the indicated restriction enzyme and then Southern blot detection of differentially sized DNA fragments (the genomic region used as probe is shown). Asterisks indicate the clonal cell lines used for subsequent studies. (B) Cells were fixed at the 9-h time point of conjugation and stained with DAPI. Each conjugating cell pair (indicated by paired arrowheads) represents the predominant state of nuclear differentiation observed in wild-type cells (top panels) or Rsp1 knockout cells (bottom panel). In wild-type cell pairs, the two developing zygotic macronuclei stain lightly, the parental macronucleus stains strongly, and two smaller micronuclear-content genomes are also evident. Cell pairs lacking parental Rspl retain the parental macronucleus. An unpaired cell with a single macronucleus and single micronucleus is indicated by an asterisk. All panels are shown at the same scale; scale bar, $10 \mu \mathrm{m}$.

clear RSP1 knockout accumulated with a mid-cell parental macronucleus and several smaller, presumably micronuclearcontent zygotic nuclei (Fig. 4B, bottom panel). This stage of conjugation arrest is similar to that observed upon mating $R D F 1$ or $R D N 2$ macronuclear gene knockouts (Lee et al. 2009). However, at low frequency, occasional RSP1 macronuclear knockout cell pairs appeared to progress past this early conjugation arrest. We note that loss of Rsp1 could impose a conjugation defect indirectly through an impact on the stability and/or function of Rdf1, Rdn1, and/or Rdn2.

\section{Accumulation of strand-specific antisense sRNAs requires both Rdr1 and Rsp1}

The high abundance of 23- to 24-nt sRNAs in growing Tetrahymena allows their direct visualization by filtrationbased size enrichment, denaturing gel electrophoresis, and SYBR Gold staining (Lee and Collins 2006). To investigate whether the loss of Rsp1 function influences the accumulation of these Dcr2-generated sRNAs, we analyzed sizeselected RNA from strains lacking Rsp1 or various RDRC subunit(s). In parallel we analyzed size-selected RNA from the corresponding wild-type strain and a strain that inducibly overexpressed catalytic-dead $\operatorname{Rdr} 1$ (D1004A) in competition with endogenous Rdr1 (Lee and Collins 2007). Surprisingly, cells lacking Rsp1 also lacked abundant 23- to 24-nt sRNAs (Fig. 5, cf. lanes 1 and 2). This loss was even more striking than the depletion of 23- to 24-nt sRNAs in any individual or combination knockout of the nonessential RDRC subunits (Southern blots [data not shown] and sRNA phenotypes [see below] confirm the knockout genotypes) (Fig. 5, lanes 4-8). Overexpression of catalytic-dead Rdr1 was also effective in depleting 23- to 24-nt sRNAs, perhaps with an increase in the general RNA fragment background (mature tRNA provides a control for RNA integrity and relative loading) (Fig. 5, lane 3).

We next used blot hybridization with individual sRNAcomplementary oligonucleotides to detect representative members of each abundant Tetrahymena class of sRNA (Fig. 5, lower panels), based on previous deep sequencing and blot hybridization data (Couvillion et al. 2009). As expected from the depletion of bulk 23- to 24-nt sRNAs, cells lacking Rsp1 or expressing catalytic-dead Rdr1 were missing the sRNA classes associated with Twi2. These sRNA classes include the pseudogene loci that generate sRNA dependent on the Rdn2 RDRC (e.g., the IIIB locus), phased cluster loci that generate sRNA dependent on the Rdf2 RDRC (e.g., the PH3 locus), and loci that generate sRNA dependent on more than one type of RDRC (e.g., the IB locus and high-copy repeat RPT1). Of note, a primary transcript capable of extensive secondary structure formation still generated processed RNA even in Rsp1 knockout cells or cells expressing catalytic-dead Rdr1 (Fig. 5, STR1), although with a broadened sRNA size distribution. This population of sRNA was most enriched in association with nuclear Twi8 rather than cytoplasmic Twi2 (Couvillion et al. 2009) and could be generated directly by Dcr2 cleavage of an annealed dsRNA hairpin.

Coincident with the loss of phased-cluster sRNAs in Rdf2 knockout strains, longer transcripts that could be Rdf2 RDRC precursors become detectable (Couvillion et al. 2009). For the PH3 locus, this longer transcript migrates as 


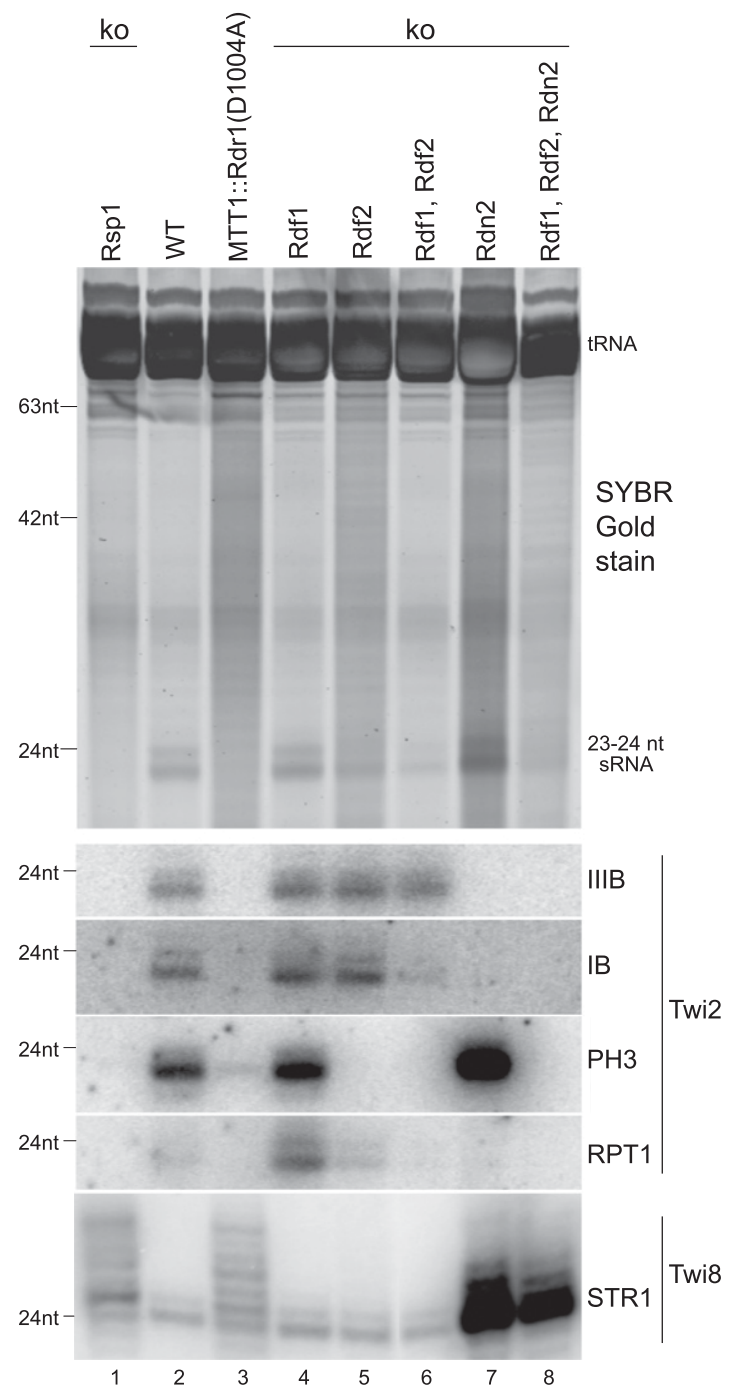

FIGURE 5. Rdr1-dependent sRNA accumulation requires Rsp1. Sizeselected RNA from the indicated strains was analyzed by denaturing PAGE and SYBR Gold stain (top panel). Northern blot hybridizations were performed with probes antisense to sRNAs IIIB, IB, PH3, RPT1, and STR1 (bottom panels), which were previously sequenced in enriched association with Twi2 or Twi8 as indicated. The same blot was probed for all sRNAs except for STR1, for which a separate blot containing the same RNAs was probed.

$>500 \mathrm{nt}$ when detected by mRNA Northern blot (Fig. 6A). To investigate the fate of a putative RDRC substrate in Rsp1 knockout cells, we first defined transcripts from the $\mathrm{PH} 3$ locus at a molecular level using RT-PCR. Transcripts contained the two regions that give rise to clustered sRNAs, joined by mRNA splicing and followed by a previously predicted 163-nt stem-loop and at least $300 \mathrm{nt}$ of sequence beyond the structured region (Fig. 6B). Transcript sequences were readily amplified by PCR after reverse transcription with $\mathrm{dT}_{18}$ primer, which suggests that at least some of the transcript population becomes polyadenylated. Because RDRC initiation requires a free single-stranded RNA $3^{\prime}$ end (Talsky and Collins 2010), the direct precursor for RDRC
dsRNA synthesis may be generated by endonucleolytic cleavage of the polyadenylated transcript (Fig. 6B).

To investigate potential RSPC involvement in the generation of an RDRC precursor, we probed for $\mathrm{PH} 3$ transcript in cells lacking Rsp1. In contrast to cells lacking Rdf2, cells lacking Rsp1 did not accumulate an mRNA-sized PH3 transcript in either of two strain backgrounds (Fig. 6A, cf. lanes 3,4 and 7,8). Interestingly, the mRNA-like PH3 transcript also did not accumulate in cells expressing catalyticdead Rdr1 (Fig. 6A, lane 8). Because cells lacking Rsp1 or expressing catalytic-dead Rdr1 also did not accumulate $\mathrm{PH} 3$

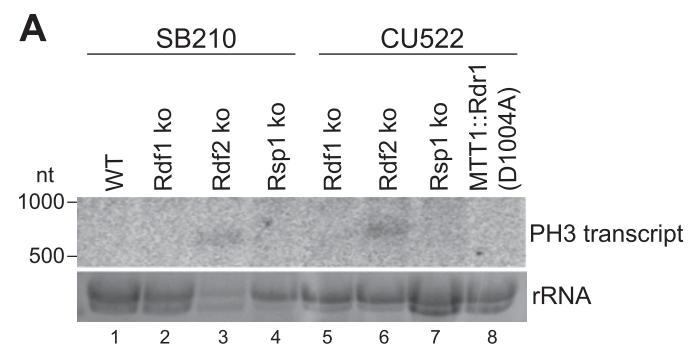

B
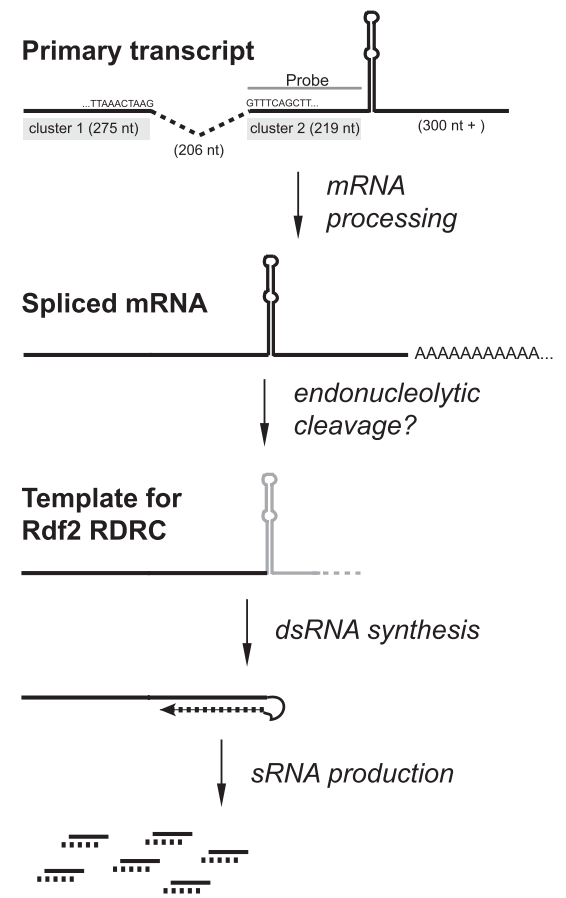

FIGURE 6. PH3 precursor transcripts do not accumulate in cells lacking Rsp1. (A) Detection of a PH3 locus transcript by Northern blot using hexamer-primed probe. The ribosomal RNA (rRNA) region of total RNA stained by ethidium bromide is shown as a loading control; note that endogenous cleavage of Tetrahymena $26 \mathrm{~S}$ rRNA creates a doublet that overlaps in migration with 17S rRNA. (B) PH3 mRNA-like transcript contains two exons (solid black lines) separated by an intron (dashed line). At least some fraction of $\mathrm{PH} 3$ transcript is processed as mRNA, based on RT-PCR detection of intron removal and polyadenylation. Further processing may occur before recognition of the transcript as an RDRC substrate, for example, a speculated endonucleolytic cleavage at the base of the stem-loop to generate a nonpolyadenylated $3^{\prime}$ end. 
sRNA, the mRNA-sized transcript may have escaped commitment to sRNA biogenesis in a manner that allowed an alternative pathway of mRNA decay. Several models for Rsp1 function can be envisioned, including potential Rsp1 activities prior to Rdr1 in RNA silencing pathways (Fig. 7; see Discussion).

\section{DISCUSSION}

Together the results above suggest that Rsp1 plays a role in RDRC-mediated sRNA biogenesis. Rsp1 assembles stable complexes with Rdn1 and an Rdf that are mutually exclusive for binding of Rdr1 and Dcr2. Consistent with the physical segregation of RSPCs and RDRCs, RSPCs lack associated RDRC activity (data not shown). However, cells lacking Rsp1 or expressing catalytic-dead Rdr1(D1004A) were similarly depleted for all known classes of Twi2-bound sRNAs. Curiously, Rdn1 uridylyltransferase activity was markedly reduced when bound to Rsp1 compared with Rdr1. This inhibition could be important for limiting Rdn1 activity in vivo.

Models for Rsp1 function include potential roles before or after dsRNA synthesis. RSPCs could select RNAs for

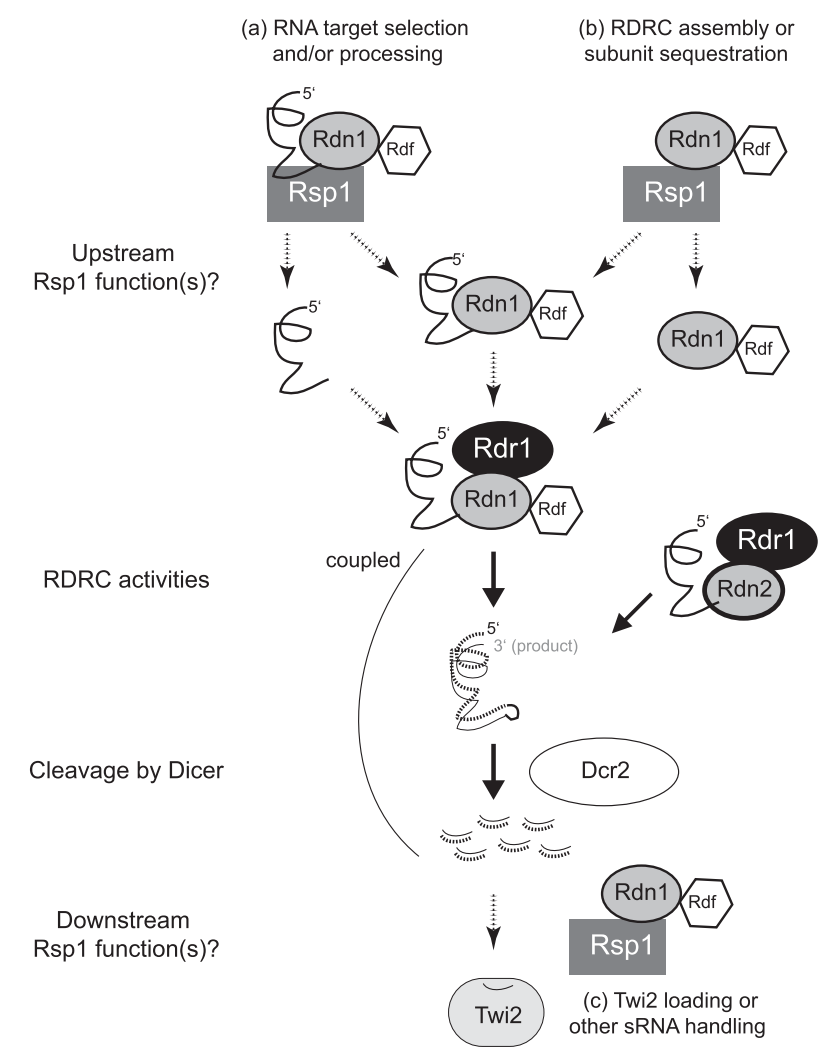

FIGURE 7. Models for Rsp1 function in RDRC-dependent sRNA biogenesis. Hypothetical Rspl functions (dashed arrows) are suggested in the context of known pathway steps (solid black arrows). For description of the three models indicated as $a, b$, and $c$, see the Discussion. commitment to an RDRC pathway (Fig. 7, model a). RNA selection or processing by an RSPC-associated activity, for example, an mRNA endonuclease, could provide the template specificity that an RDRC itself may lack. The subunit sharing between RSPCs and RDRCs may directly or indirectly facilitate RNA handoff. Alternately, RSPCs could maintain a cellular reservoir of $\operatorname{Rdn} 1 / \operatorname{Rdf}$ complexes that is sequestered from Rdr1 or that promotes stable Rdn1/Rdf folding prior to RDRC assembly (Fig. 7, model b). The models above do not necessarily predict the observed influence of Rsp1 loss of function on Rdn2 RDRC sRNAs. Instead of reflecting a loss of function, the disruption of Rdn2dependent pseudogene loci sRNA biogenesis could reflect a dominant-negative impact of altered RNA silencing. In an entirely different type of model, Rsp 1 could act subsequent to RDRC-mediated dsRNA synthesis and cotranscriptional cleavage by Dcr2 (Fig. 7, model c). For example, RSPCs could modify sRNAs or mediate sRNA loading to Twi2, thereby promoting sRNA stabilization as RNP. However, if RSPC function is downstream from Dcr2, it is not obvious why RSPCs and RDRCs would share Rdn1 and Rdf subunits.

To our surprise, we found that unlike Rdr1 and Rdn1 (Lee and Collins 2007; Lee et al. 2009), Rsp1 is not essential for vegetative growth. The corresponding viability of cells lacking any known Twi2-bound sRNA suggests that production of these sRNAs may be physiologically most significant as the degradation end-point for nonfunctional transcripts rather than as a mechanism of post-transcriptional gene silencing. This would be consistent with our observations that some of the 23- to 24-nt sRNA biogenesis machinery have a genetically essential role in conjugation rather than vegetative growth (Lee et al. 2009). The need for Rdn1, Rdr1, and Dcr2 function in vegetative growth could reflect a requirement to prevent the accumulation of toxic intermediates of sRNA biogenesis. Aberrantly accumulating mRNA or mRNA-like transcripts could overwhelm the cellular machinery for RNA surveillance and degradation, or accumulating dsRNAs could constitutively activate viral defense (Howard-Till and Yao 2006).

The apparently RDRC-independent biogenesis of at least one class of Twi8-bound sRNAs suggests that these sRNAs could be generated by direct Dcr2 cleavage, uncoupled from dsRNA synthesis by Rdr1. Curiously, in vitro Dcr2 cleavage of annealed dsRNA can generate an increased heterogeneity of sRNA lengths compared to cleavage that is cotranscriptional with dsRNA synthesis (Lee and Collins 2007). Correspondingly, cells lacking Rsp1 or expressing catalytic-dead Rdr1(D1004A) have an increased heterogeneity of STR1 sRNAs. The more uniform size distribution of STR1 sRNAs in wild-type cells suggests that some transcripts from loci that generate Twi8-bound sRNAs are normally directed to RDRC-mediated dsRNA synthesis and cotranscriptional Dcr2 cleavage. Twi8 is not essential in at least some strain backgrounds (Couvillion et al. 2009), but it remains a possibility 
that some loci can produce essential 23- to 24-nt sRNAs by more than one biogenesis pathway.

\section{MATERIALS AND METHODS}

\section{Cell lines and culture conditions}

Integration constructs for protein tagging fused the C-terminal amino acid of the endogenous open reading frame to the epitope $\operatorname{tag}(\mathrm{s})$, followed by the RPL29 $3^{\prime}$ untranslated region (UTR) and a Neo2 cassette with the BTU2 3' UTR. A protease cleavage site separated the tandem Protein A module from the remainder of the polypeptide to allow elution from IgG resin (see below). Integration constructs for gene knockout replaced the protein open reading frame with the Bsr2 cassette (MTT1 promoter, open reading frame encoding resistance to blasticidin, BTU2 3' UTR). Cells were selected for integration and assortment of $\mathrm{Neo} 2$ using paromomycin or for integration of Bsr2 using blasticidin with $0.7 \mu \mathrm{g} / \mathrm{mL} \mathrm{CdCl}_{2}$ followed by assortment in blasticidin without $\mathrm{CdCl}_{2}$ to reduce cassette expression.

Cultures were grown shaking at $30^{\circ} \mathrm{C}$ in NEFF $(0.5 \%$ proteose peptone, $0.5 \%$ yeast extract, $1 \%$ glucose, $10 \mu \mathrm{M} \mathrm{FeCl}_{3}$ ) and harvested for extract production at a density of $2 \times 10^{5}$ to $5 \times 10^{5}$ cells $/ \mathrm{mL}$. For zz-Rdr1(D1004A) expression (Lee and Collins 2007), cells were inoculated at $1 \times 10^{4}$ to $2 \times 10^{4}$ cells $/ \mathrm{mL}$ and grown for 16-20 h in the presence of $1 \mu \mathrm{g} / \mathrm{mL} \mathrm{CdCl}_{2}$. For conjugation, cells were starved in $10 \mathrm{mM}$ Tris- $\mathrm{HCl}(\mathrm{pH} 7.5)$, mixed, and incubated at $30^{\circ} \mathrm{C}$ without shaking. At least 200 cell pairs of each genotype were counted to score the stage of nuclear differentiation. Cells were fixed in $2 \%$ paraformaldehyde for $1 \mathrm{~h}$, washed twice in modified PBS $(130 \mathrm{mM}$ $\mathrm{NaCl}, 2 \mathrm{mM} \mathrm{KCl}, 8 \mathrm{mM} \mathrm{Na}_{2} \mathrm{HPO}_{4}, 2 \mathrm{mM} \mathrm{KH}_{2} \mathrm{PO}_{4}, 10 \mathrm{mM}$ ethylene glycol tetra-acetic acid at $\mathrm{pH} 7.2$ ) with $0.1 \%$ bovine serum albumin (Sigma), incubated in $0.1 \mu \mathrm{M}$ DAPI for $10 \mathrm{~min}$, washed once more in modified PBS, and mounted in modified PBS with 70\% glycerol.

\section{Protein purification and activity assays}

Proteins were purified by binding to IgG agarose and/or M2 Flag antibody resin using previously developed cell lysis and purification conditions (Lee and Collins 2007). Resin-bound complexes were washed in $200 \mathrm{mM} \mathrm{NaCl}$, except for the low-salt washes of $50 \mathrm{mM} \mathrm{NaCl}$ as indicated. IgG agarose elution was performed by addition of Tobacco Etch Virus (TEV) protease; Flag antibody elution was performed using triple Flag peptide (Sigma). Uridylyltransferase activity was assayed using a 79-nt single-stranded RNA1 template and radiolabeled UTP diluted to a final concentration of $20 \mu \mathrm{M}$ (Talsky and Collins 2010). To prevent RNA secondary structure formation during gel electrophoresis, radiolabeled products were analyzed by highly denaturing formamide-PAGE (9\%-12\% of 19:1 acrylamide:bis-acrylamide, $7 \mathrm{M}$ urea, $45 \%$ formamide, $1 \times \mathrm{TBE})$.

\section{RNA purification and detection}

RNA isolation, sRNA enrichment, and blot hybridization methods were as previously described (Couvillion et al. 2009; Lee and Collins 2006). Total RNA was extracted using TRIzol reagent (Invitrogen) from cells washed into $10 \mathrm{mM}$ Tris- $\mathrm{HCl}$ ( $\mathrm{pH}$ 7.5). Enrichment for sRNA was done using YM50 Microcon columns (Amicon). Blot hybridization for sRNAs after acrylamide gel electrophoresis used end-labeled DNA oligonucleotides, while hybridization for mRNA Northern blots and genomic DNA Southern blots used hexamerlabeled double-stranded DNA probes. Oligonucleotide probe sequences were complementary to previously sequenced sRNAs: IIIB (AGCAAAGACGATTAACAATATTCA), IB (ATTTACTAGATGT ATTTCCCTTA), PH3 (AACTATTTAATAATATATTTTCA), RPT1 (AATATCACAATCCAAAACAAATA), and STR1 (AAGCTTCTC TTATCTTCTAATCA). The mRNA-sized PH3 transcript was analyzed by RT-PCR from total RNA of CU522 wild-type and Rdf2 knockout cells after treatment with RQ1 RNase-free DNase (Promega).

\section{DATA DEPOSITION}

The GenBank accession number for RSP1 is JQ797583.

\section{ACKNOWLEDGMENTS}

We thank Mary Couvillion for reagents, discussion, and comments on the manuscript.

Received March 28, 2012; accepted May 22, 2012.

\section{REFERENCES}

Bouhouche K, Gout JF, Kapusta A, Betermier M, Meyer E. 2011. Functional specialization of Piwi proteins in Paramecium tetraurelia from post-transcriptional gene silencing to genome remodelling. Nucleic Acids Res 39: 4249-4264.

Carthew RW, Sontheimer EJ. 2009. Origins and mechanisms of miRNAs and siRNAs. Cell 136: 642-655.

Couvillion MT, Lee SR, Hogstad B, Malone CD, Tonkin LA, Sachidanandam R, Hannon GJ, Collins K. 2009. Sequence, biogenesis, and function of diverse small RNA classes bound to the Piwi-family proteins of Tetrahymena thermophila. Genes Dev 23: 2016-2032.

Duharcourt S, Lepere G, Meyer E. 2009. Developmental genome rearrangements in ciliates: a natural genomic subtraction mediated by non-coding transcripts. Trends Genet 25: 344-350.

Grewal SI. 2010. RNAi-dependent formation of heterochromatin and its diverse functions. Curr Opin Genet Dev 20: 134-141.

Howard-Till RA, Yao MC. 2006. Induction of gene silencing by hairpin RNA expression in Tetrahymena thermophila reveals a second small RNA pathway. Mol Cell Biol 26: 8731-8742.

Ketting RF. 2011. The many faces of RNAi. Dev Cell 20: 148-161.

Lee SR, Collins K. 2006. Two classes of endogenous small RNAs in Tetrahymena thermophila. Genes Dev 20: 28-33.

Lee SR, Collins K. 2007. Physical and functional coupling of RNAdependent RNA polymerase and Dicer in the biogenesis of endogenous siRNAs. Nat Struct Mol Biol 14: 604-610.

Lee SR, Talsky KB, Collins K. 2009. A single RNA-dependent RNA polymerase assembles with mutually exclusive nucleotidyl transferase subunits to direct different pathways of small RNA biogenesis. RNA 15: 1363-1374.

Malone CD, Anderson AM, Motl JA, Rexer CH, Chalker DL. 2005. Germ line transcripts are processed by a Dicer-like protein that is essential for developmentally programmed genome rearrangements of Tetrahymena thermophila. Mol Cell Biol 25: 9151-9164.

Moazed D. 2011. Mechanisms for the inheritance of chromatin states. Cell 146: $510-518$.

Mochizuki K. 2012. Developmentally programmed, RNA-directed genome rearrangement in Tetrahymena. Dev Growth Differ 54: $108-119$. 
Mochizuki K, Gorovsky MA. 2005. A Dicer-like protein in Tetrahymena has distinct functions in genome rearrangement, chromosome segregation, and meiotic prophase. Genes Dev 19: 77-89.

Pak J, Fire A. 2007. Distinct populations of primary and secondary effectors during RNAi in C. elegans. Science 315: 241-244.

Sijen T, Steiner FA, Thijssen KL, Plasterk RH. 2007. Secondary siRNAs result from unprimed RNA synthesis and form a distinct class. Science 315: 244-247.

Talsky KB, Collins K. 2010. Initiation by a eukaryotic RNA-dependent RNA polymerase requires looping of the template end and is influenced by the template-tailing activity of an associated uridyltransferase. J Biol Chem 285: 27614-27623.

Thivierge C, Makil N, Flamand M, Vasale JJ, Mello CC, Wohlschlegel J, Conte D Jr, Duchaine TF. 2012. Tudor domain ERI-5 tethers an RNA-dependent RNA polymerase to DCR-1 to potentiate endoRNAi. Nat Struct Mol Biol 19: 90-97.

Vazquez F, Vaucheret H, Rajagopalan R, Lepers C, Gasciolli V, Mallory AC, Hilbert JL, Bartel DP, Crété P. 2004. Endogenous trans-acting siRNAs regulate the accumulation of Arabidopsis mRNAs. Mol Cell 16: 69-79.

Voinnet O. 2008. Use, tolerance and avoidance of amplified RNA silencing by plants. Trends Plant Sci 13: 317-328. 

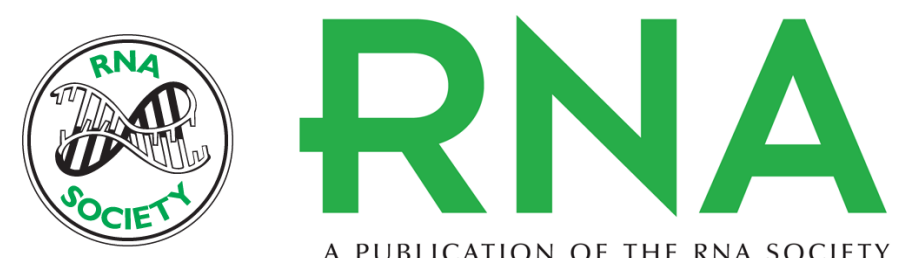

A PUBLICATION OF THE RNA SOCIETY

\section{Strand-asymmetric endogenous Tetrahymena small RNA production requires a previously uncharacterized uridylyltransferase protein partner}

Kristin Benjamin Talsky and Kathleen Collins

RNA 2012 18: 1553-1562 originally published online June 15, 2012

Access the most recent version at doi:10.1261/rna.033530.112

References This article cites 20 articles, 9 of which can be accessed free at:

http://rnajournal.cshlp.org/content/18/8/1553.full.html\#ref-list-1

\section{License}

Email Alerting Receive free email alerts when new articles cite this article - sign up in the box at the Service top right corner of the article or click here.

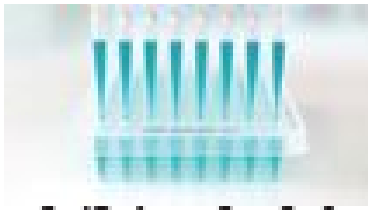

Providing Precise Solutions for your research.

To subscribe to RNA go to:

http://rnajournal.cshlp.org/subscriptions 